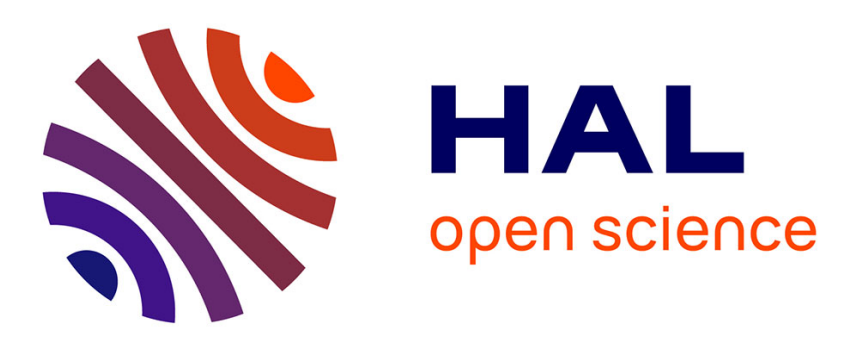

\title{
Spectral broadening of picosecond pulses forming dispersive shock waves in optical fibers
}

Alexandre Parriaux, Matteo Conforti, A. Bendahmane, Julien Fatome, Christophe Finot, Stefano Trillo, Nathalie Picqué, Guy Millot

\section{To cite this version:}

Alexandre Parriaux, Matteo Conforti, A. Bendahmane, Julien Fatome, Christophe Finot, et al.. Spectral broadening of picosecond pulses forming dispersive shock waves in optical fibers. Optics Letters, 2017, 42 (15), pp.3044-3047. 10.1364/OL.42.003044 . hal-01563643

\section{HAL Id: hal-01563643 \\ https://hal.science/hal-01563643}

Submitted on 17 Jul 2017

HAL is a multi-disciplinary open access archive for the deposit and dissemination of scientific research documents, whether they are published or not. The documents may come from teaching and research institutions in France or abroad, or from public or private research centers.
L'archive ouverte pluridisciplinaire HAL, est destinée au dépôt et à la diffusion de documents scientifiques de niveau recherche, publiés ou non, émanant des établissements d'enseignement et de recherche français ou étrangers, des laboratoires publics ou privés. 


\title{
Spectral broadening of picosecond pulses forming dispersive shock waves in optical fibers
}

\author{
A. Parriaux, ${ }^{1}$ M. Conforti, ${ }^{2}$ A. Bendahmane, ${ }^{1}$ J. Fatome, ${ }^{1}$ C. Finot, ${ }^{1}$ S. \\ Trillo, ${ }^{3}$ N. Picque,,$^{4,5}$ ANd G. Millot ${ }^{1, *}$ \\ ${ }^{1}$ Laboratoire Interdisciplinaire Carnot de Bourgogne (ICB), UMR6303 CNRS-Univ. Bourgogne Franche Comté, Dijon, France. \\ ${ }^{2}$ Laboratoire de Physique des Lasers Atomes et Molécules (PhLAM), UMR8523 CNRS-Univ. Lille, Lille, France. \\ ${ }^{3}$ Department of Engineering, University of Ferrara, Via Saragat 1, 44122 Ferrara, Italy. \\ ${ }^{4}$ Max-Planck-Institut für Quantenoptik (MPQ), Garching, Germany. \\ ${ }^{5}$ Institut des Sciences Moléculaires d'Orsay (ISMO), UMR8214 CNRS-Univ. Paris-Sud, Orsay, France. \\ *Corresponding author: Guy.Millot@u-bourgogne.fr
}

Received XX Month XXXX; revised XX Month, XXXX; accepted XX Month XXXX; posted XX Month XXXX (Doc. ID XXXXX); published XX Month XXXX

We investigate analytically, numerically and experimentally the spectral broadening of pulses that undergo the formation of dispersive shocks, addressing in particular pulses in the range of tens of ps generated via electro-optic modulation of a continuous-wave laser. We give an analytical estimate of the maximal spectral extension and show that super-Gaussian waveforms favor the generation of flat-topped spectra. We also show that the weak residual background of the modulator produces undesired spectral ripples. Spectral measurements confirm our estimates and agree well with numerical integration of the nonlinear Schrödinger equation.

OCIS codes: (190.4370) Nonlinear optics, fibers; (190.5530) Pulse propagation and temporal solitons.

Dispersive shock waves (DSWs) or undular bores are expanding nonlinear wave packets composed of adjacent rapid oscillations slowly modulated in amplitude and frequency, which have been investigated in different physical contexts [1-4]. Coherent DSWs develop in homogeneous or disordered media exhibiting strong nonlinearity, weak dispersion and negligible dissipation. In such media the shock waves originates from a wave-breaking process or "gradient catastrophe" regularized by dispersive effects. In optical fibers such wave-breaking have been observed long ago in the normal dispersive regime when self-phase modulation (SPM) dominates [5-7]. More recently such regime has conveyed a renewed interest, considerably extending the importance of fiber DSWs [8-15]. The main signature of DSWs is the appearance of temporal ondulations near the pulse edges that induce spectral sidelobes. The deleterious effect of these inherent temporal oscillations can be avoided in practice with the use of non-breaking parabolic pulses [16, 17]. However, DSWs can be judiciously exploited to generate smooth and coherent continuum spectra in normally dispersive nonlinear fibers [9]. A particularly interesting application of DSW-induced spectral broadening is the generation of flat-topped low noise frequency combs obtained by electro-optic (e-o) intensity modulation of a continuous-wave (cw) laser. The shape of the spectral envelope of such combs plays a crucial role in applications such as direct frequency comb spectroscopy $[18,19]$, radio-frequency photonics [20,21] or telecommunications [22]. But because of the limited extinction ratio of high-bandwidth e-o modulators (typically $30 \mathrm{~dB}$ ) the pulse train is superimposed on a residual continuous background. Even a weak background strongly enhances the extension and the contrast of the temporal oscillations inherent to the dispersive shock [14, 15, 23].

In this letter, we address the study of DSW-induced spectral broadening of 50-ps pulses. We evaluate the influence of the pulse shape and assess the impact of the residual background. We show that super-Gaussian pulses are best suited to generate smooth and flat-topped spectra. We propose an analytical estimate of the maximum spectral extension of the DSW, which mainly depends on the fiber parameters and input pulse peak power, but depends only slightly on the shape and duration of the input pulses. Experimental measurements carried out for different propagation distances or different input powers quantitatively confirm the analytical estimate of the pulse spectral extension and are in excellent agreement with numerical integration of the nonlinear Schrödinger equation (NLSE).

The propagation of an envelope wave $A$ in an optical fiber is governed by the NLSE which can be written in dimensional form as:

$$
i \frac{\partial A}{\partial z}-\frac{1}{2} \beta_{2} \frac{\partial^{2} A}{\partial t^{2}}+\gamma|A|^{2} A+i \frac{\alpha}{2} A=0
$$


where $\gamma$ is the nonlinear coefficient, $\beta_{2}$ is the second-order chromatic dispersion coefficient and $\alpha$ is the linear loss. $z$ is the propagation distance and $t$ a local time in a frame of reference which moves at the group velocity. In the NLSE $|A|^{2}=P$ is the wave power. We focus our attention here on the case of pulses generated from a cw laser, intensity modulated by an electro-optic modulator (EOM). Because any real EOM has a finite extinction ratio (ER) the pulse train always contains a cw background or leakage. The factor ER can be defined as ER $=\left(P+P_{o}\right) / P_{o}$ where $P$ and $P_{o}$ are the pulse peak-power and cw background power of the modulated light, respectively. The light modulated by a nonideal EOM can be seen as the sum of a pulse without cw component and a leakage light. Following the procedure described in refs. [24-25] taking into account the finite ER of the intensity modulator and neglecting loss, Eq. (1) possesses the following conservation law

$$
\frac{\mathrm{d}}{\mathrm{d} z}\left(\gamma \frac{\int\left(|A(z, t)|^{2}-P_{o}\right)^{2} \mathrm{~d} t}{2 \int\left(|A(z, t)|^{2}-P_{o}\right) \mathrm{d} t}+\beta_{2} \frac{\int \omega^{2}\left(|\tilde{A}(z, \omega)|^{2}-P_{o} \delta(\omega)\right) \mathrm{d} \omega}{2 \int\left(|\tilde{A}(z, \omega)|^{2}-P_{o} \delta(\omega)\right) \mathrm{d} \omega}\right)=0 .
$$

The two terms of Eq. (2) have a dimension of an inverse length, and can be considered as a generalization of the inverse of the standard nonlinear and dispersion lengths [24]. In practice, for a pulse propagating in the highly nonlinear regime, only the first integral will be relevant; conversely in the purely dispersive regime only the second integral matters. Equation (2) can be rewritten as [24]:

$$
\mathcal{L}_{N L}^{-1}(z)+\mathcal{L}_{D}^{-1}(z)=C,
$$

where $C$ is a constant fixed by the input conditions.

Here, in the general case the input conditions correspond to an e-o wave consisting of 50-ps pulses of peak power $P$ and normalized power profile $f(t)$ superimposed on a residual continuous background of power $P_{o}$. The e-o wave propagates through a highly dispersive single mode fiber with $\beta_{2}=0.119 \mathrm{ps}^{2} \mathrm{~m}^{-1}$ and $\gamma=4.6 \times 10^{-3} \mathrm{~W}^{-1} \mathrm{~m}^{-1}$ at a carrier wavelength of $1560 \mathrm{~nm}$.

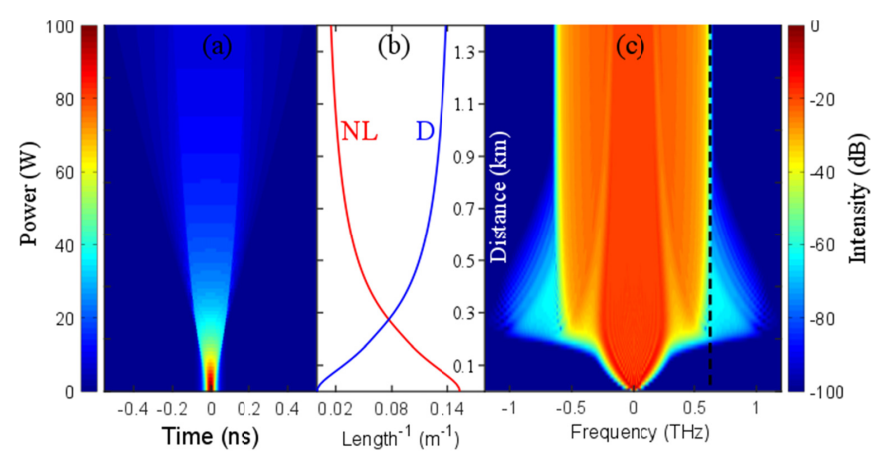

Fig. 1. (a) Temporal and (c) spectral color map evolution vs propagation distance for a 50-ps hyperbolic secant pulse with $P=$ $100 \mathrm{~W}$ and without background $\left(P_{o}=0\right)$. The dashed line shows the maximum spectral extension predicted by Eq. (8). (b) Evolution of $\mathcal{L}_{N L}^{-1}$ and $\mathcal{L}_{D}^{-1}$ versus propagation distance.
Figure 1 shows the temporal (a) and spectral (c) evolutions along the propagation distance in the particular case of hyperbolic secant pulses without background or loss. With such conditions, one can distinguish two stages of the pulse evolution. As can be seen from Fig. 1, at the early stage of propagation, the nonlinear effect, i.e. self-phase modulation, dominates, thus leading to a significant spectral broadening $\frac{\mathcal{L}_{N L}^{-1}(z=0)}{\mathcal{L}_{D}^{-1}(z=0)} \cong 6200$ corresponding to an equivalent soliton number $\mathrm{N}=52$ [9]). Subsequently, for larger propagation distances, this resulting spectral expansion, associated with a strong steepening of the pulse edges, makes dispersion to acquire weight. At the shock distance $\mathrm{z}_{\mathrm{c}}=274 \mathrm{~m}$ (see Fig. 1(b)), nonlinearity and dispersion have approximately the same importance $\left(\mathcal{L}_{N L}^{-1}=\mathcal{L}_{D}^{-1}\right)$ and sidelobes appear in the spectrum. After the shock point, the pulse disperses and its peak power decreases, making the evolution progressively almost linear. In this second regime dispersion dominates and the spectrum is almost conserved while the pulse strongly temporally broadens. This process can be summarized by help of Eq. (3) as follows [24]:

$$
\mathcal{L}_{N L}^{-1}(0) \approx \mathcal{L}_{D}^{-1}\left(z \gg z_{c}\right),
$$

where $z \gg Z_{c}$ means at a distance well beyond the shock point. In Eq. (2) we can recognize the variance of the power spectrum $\sigma_{\omega}^{2}$ deprived of the cw component defined as

$$
\sigma_{\omega}^{2}=\frac{\int \omega^{2}|\tilde{A}(z, \omega)|^{2} \mathrm{~d} \omega}{\int|\tilde{A}(z, \omega)|^{2} \mathrm{~d} \omega-P_{o}} .
$$

From Eqs. (2), (4) and (5) and with an input condition $|A(0, t)|^{2}=P_{o}+P f(t)$ the root mean square (rms) $\sigma_{\omega}$ at a propagation distance well beyond the wavebreaking point can be expressed as

$$
\sigma_{\omega}=\sqrt{\frac{\gamma P}{\beta_{2}}} F
$$

where the dimensionless factor $F$ is defined as

$$
F^{2}=\frac{\int f(t)^{2} \mathrm{~d} t}{\int f(t) \mathrm{d} t} .
$$

The parameter $F$ does not depend on the background level and on the input pulse duration and can be calculated explicitly for different input temporal profiles. The factor $F$ is approximately equal to one for relevant pulses. For example $F=2^{-1 / 4 m}$ for a super-Gaussian of order $m$ (e.g. $F$ $=0.944$ for $m=3$ ). Consequently, considering that most of the spectrum ranges in the interval $\left[-2 \sigma_{\omega} ; 2 \sigma_{\omega}\right]$, then the maximum of spectral extension $\omega_{\max }$ can be calculated as

$$
\omega_{\max } \sqrt{\frac{\beta_{2}}{\gamma P}}=2 \sigma_{\omega} \sqrt{\frac{\beta_{2}}{\gamma P}}=2 F \cong 2 .
$$

Equation (8) shows that spectral extension depends only slightly on the pulse profile but is independent of the pulse duration and scales as $\sqrt{\gamma P / \beta_{2}}$ corresponding to the inverse of the average temporal period of the DSWs obtained from Whitham modulation theory $[1,11,15]$. This scaling is identical to that predicted for parabolic nonlineardispersive similaritons of passive fibers [26]. Note that for a constant pulse peak power $P$ the maximum spectral extension does not depend on the power level $P_{o}$ of the continuous background. 


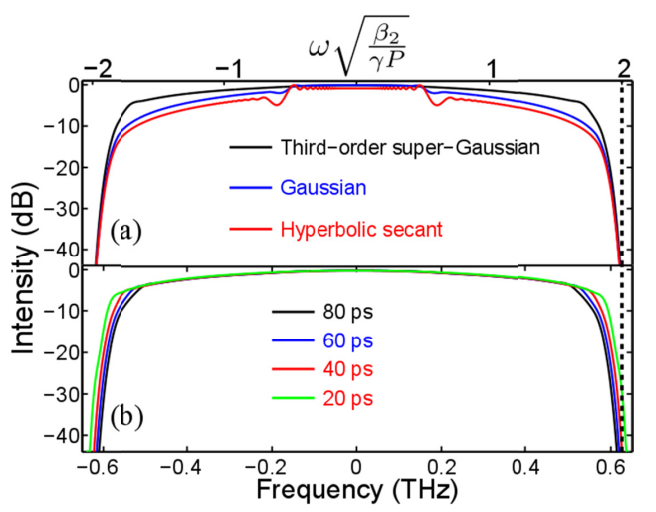

Fig. 2. (a) Variation of spectral broadening at the fiber output vs input temporal profile for 50 ps pulses with the same peak power $P$; (b) Spectral broadening obtained for third-order super-Gaussian of different pulse durations. Here the input pulse power is $P=100 \mathrm{~W}$ and the background is zero $\left(P_{o}=0\right)$. The dashed lines show the maximum spectral extension predicted by Eq. (8).

These remarkable properties have been verified by numerical integration of the NLSE (1) without losses as shown in Fig. 2. Figure 2(a) shows the simulated spectral profiles obtained at the fiber output for three different input profiles of identical duration and pulse peak power $P$. We can see that the same spectral extension is obtained for the three profiles but that super-Gaussian pulses lead to the flattest spectrum. Figure 2(b) confirms, in the case of superGaussian pulses, that the spectral extension depends very little on the pulse duration over a large time scale from 20 ps to 80 ps. A good agreement is obtained with the maximum spectral extension predicted by Eq. (8) (dashed vertical lines). This latter remarkable property is also verified for other input pulse profiles and for initially chirped pulses. Note that the factor 2 in Eq. (8) is in good agreement with the fact that, well beyond the shock, the pulse spectrum becomes nearly parabolic [27] with a maximum extension equal to its rms width multiplied by $\sqrt{5} \cong 2.2$. More precisely an input parabolic pulse, for which $F=2 / \sqrt{5}$, leads to a parabolic output spectrum with a maximum extension defined by $\omega_{\max } \sqrt{\frac{\beta_{2}}{\gamma P}}=\frac{2 \sqrt{5}}{\sqrt{5}}=2$. Another interesting feature is that a small continuous component due to the non-ideal EOM is sufficient to induce strong distortions of the temporal [11] and spectral profiles as visible in Figs. 3(c)-(f). Thus, in the absence of background [Figs. 3(a),(b)] both pulse and spectrum exhibit a smooth shape, while, in the presence of the latter, rapid oscillations with high contrast appear on the pulse profile as well as on the spectrum [Figs. 3(c)-(f)]. The contrast of the oscillations increases strongly with the level of the continuous background. Figure 3(f) shows that a modulator with $\mathrm{ER}=20 \mathrm{~dB}$ generates a spectrum which is not very suitable for applications, given the strong fluctuations of its intensity. Electro-optic modulators with ER of at least $30 \mathrm{~dB}$ are thus required for accurate applications [18-22]. The flatness of the spectra could be easily increased by cascading two modulators. Figure 3(a) clearly shows that the pulse acquires a parabolic profile far away the shock point as already mentioned in Ref. [27].

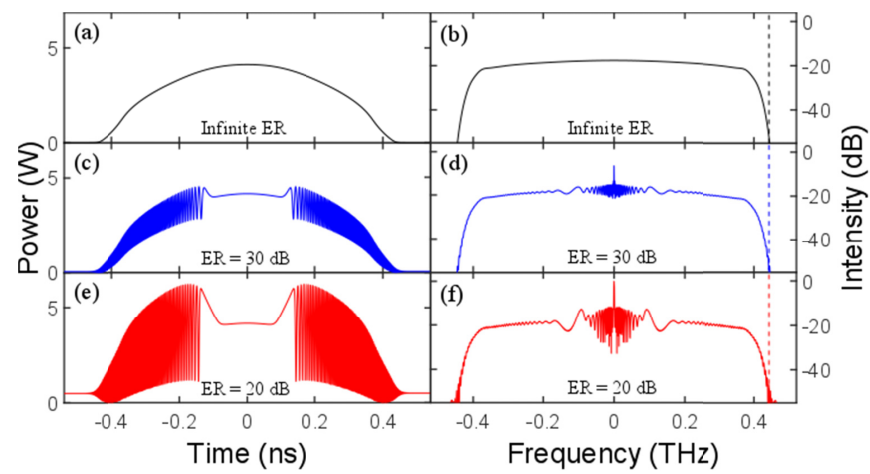

Fig. 3. Temporal and spectral profiles obtained at the fiber output from numerical integration of the NLSE (1). The input third-order super-Gaussian pulse has a peak power $P=50 \mathrm{~W}$ : (a), (b) infinite $E R$ (no background), (c), (d) ER $=30 \mathrm{~dB}$, (e), (f) ER = $20 \mathrm{~dB}$. The dashed lines show the maximum spectral extension predicted by Eq. (8).

We performed a series of experiments to illustrate the spectral broadening of the e-o pulses by the DSW process. The dispersion and nonlinear parameters of the fiber are the same as those used in the simulations and the linear loss coefficient is $\alpha=0.55 \mathrm{~dB} / \mathrm{km}$. A cw centered around $1560 \mathrm{~nm}$ is first generated by a diode laser and then intensity modulated by an EOM set at a repetition frequency of $100 \mathrm{MHz}$ and delivering 52-ps third-order superGaussian pulses [18]. Figure 4 shows the input spectrum (black curve) together with spectra recorded for different fiber lengths from $50 \mathrm{~m}$ to $1400 \mathrm{~m}$. Here the pulse peak power is $P=47 \mathrm{~W}$ and $E R=30 \mathrm{~dB}$. The input optical spectrum shows a sinc squared shape as expected for nearly-square pulses. The narrow peak at the center of the spectra is the signature of the residual $\mathrm{cw}$ component. At propagation distance of $50 \mathrm{~m}$ the spectrum expands substantially and keeps broadening significantly up to a distance of $800 \mathrm{~m}$. For further propagation distances the spectral range begins to saturate and the spectrum becomes more and more flat. Spectral oscillations appear due to the continuous background in good agreement with the numerical simulations of Fig. 3(d).

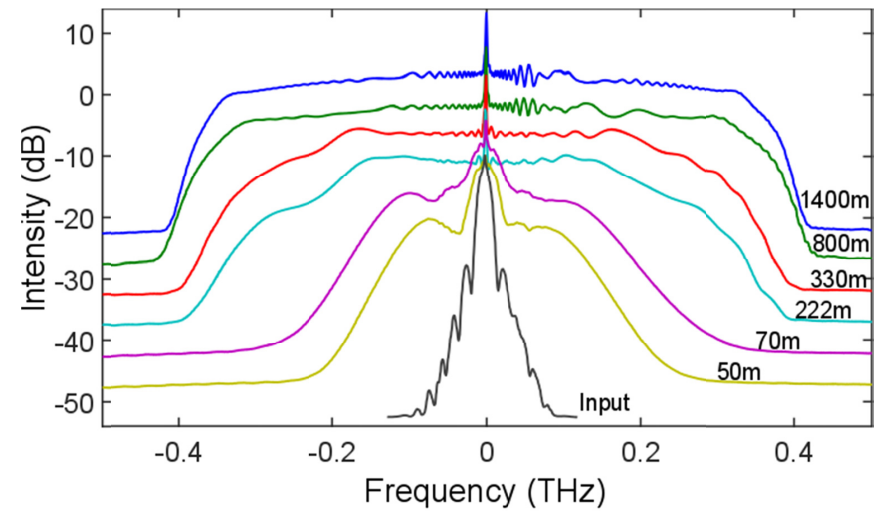

Fig. 4. Experimental spectra recorded at $1559.34 \mathrm{~nm}$ for several propagation distances ranging from $0 \mathrm{~m}$ (input) to $1400 \mathrm{~m}$. The pulse peak power is $P=47 \mathrm{~W}$ and the extinction ratio is $\mathrm{ER}=30 \mathrm{~dB}$. The curves are shifted by $5 \mathrm{~dB}$ with respect to each other for clearness. 
The spectral asymmetry originates from the slight asymmetry of the input condition (see the input spectrum with black line).

Figure 5(a) shows the variation versus propagation distance of the full width determined at $-20 \mathrm{~dB}$ of the maximum intensity (cw component excluded) of the spectra displayed in Fig. 4. We observe that the spectral width tends rapidly towards a maximum limit value corresponding to the propagation regime dominated by dispersion. But it should be noted that the widths at $-20 \mathrm{~dB}$ are indeed slightly less than the theoretical estimate given by Eq. (8). Let us note also an excellent agreement between the measurements and the spectral width determined from numerical integration of the NLSE (1) without any adjusted parameter and including losses. Figure 5(b) shows the spectral width measured as a function of the square root of input power at a propagation distance of $1400 \mathrm{~m}$. The perfect linear evolution clearly demonstrates that the spectral extension scales as $\sqrt{P}$.

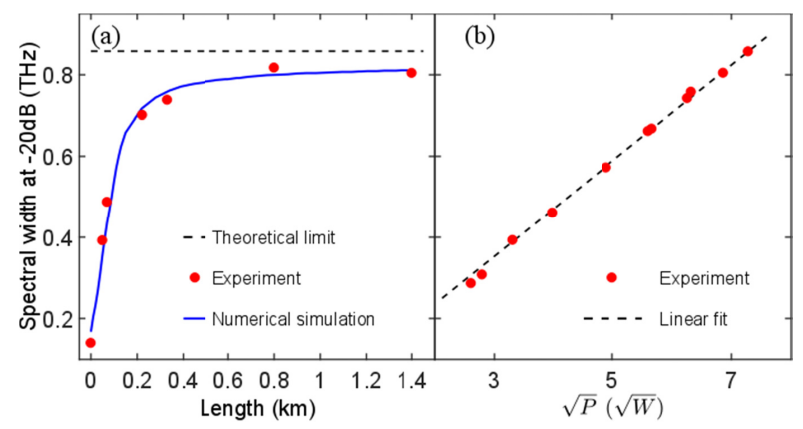

Fig. 5. (a) Variation of the full spectral width at $-20 \mathrm{~dB}$ vs propagation distance and at a fixed pulse power $P=47 \mathrm{~W}$. Same fiber parameters and pulse profile as in Fig. 4. (b) Spectral width evolution vs input pulse power at a propagation distance of $1400 \mathrm{~m}$. Same fiber parameters and pulse profile as in Fig. 4 and ER $=30 \mathrm{~dB}$.

In conclusion, we revealed the influence of the residual continuous background inherent to the finite ER of any nonideal e-o intensity modulator. We showed that a low extinction ratio results in a substantial enhancement of the contrast of the spectral oscillations inherent to DSW and is thus unacceptable for the generation of flat and smooth spectra. Well beyond the shock distance the pulses enter into a regime dominated by dispersion and the spectrum evolution becomes quasi-stationary. We analytically calculated the maximum spectral extension and showed that, at a constant pulse peak power, it does not depend on the background level and is quasi-independent of the shape of the pulses as well as of their duration. However, we demonstrated that super-Gaussian pulses are more adapted to produce flat-topped and smooth spectra than Gaussian or hyperbolic secant pulses. The experimental measurements of the spectral width as a function of propagation distance are in good agreement with the numerical integration of the NLSE and the maximum spectral extension scales as $\sqrt{P}$. We believe that our results will be very useful for the design of e-o frequency combs dedicated to various applications and relevant to many systems requiring spectral broadening of pulses of duration of tens of ps.

Funding. Conseil Régional de Bourgogne; Labex ACTION ANR11-LABX-0001-01; iXCore Research Foundation; Munich Center for Advanced Photonics.

Acknowledgment. We thank A. Mussot and S. Wabnitz for stimulating discussions.

\section{REFERENCES}

1. G. A. El and M. A. Hofer, Physica D 333, 11-65 (2016).

2. A. M. Kamchatnov, A. Gammal, and R. A. Kraenkel, Phys. Rev. A 69, 063605 (2004).

3. W. Wan, S. Jia, and J. W. Fleischer, Nat. Phys. 3, 46-51 (2007).

4. M. D. Maiden, N. K. Lowman, D. V. Anderson, M. E. Schubert, and M. A. Hoefer, Phys. Rev. Lett. 116, 174501 (2016).

5. W. J. Tomlinson, R. H. Stolen, and A. M. Johnson, Opt. Lett. 10, 457-459 (1985).

6. J. E. Rothenberg and D. Grischkowsky, Phys. Rev. Lett. 62, 531-534 (1989).

7. D. Anderson, M. Desaix, M. Lisak, and M. L. Quiroga-Teixeiro, J. Opt. Soc. Am. B. 9, 1358-1361 (1992).

8. C.-J. Rosenberg, D. Anderson, M. Desaix, P. Johannisson, and M. Lisak, Opt. Comm. 273, 272-277 (2007).

9. C. Finot, B. Kibler, L. Provost, and S. Wabnitz, J. Opt. Soc. Am. B. 25, 1938-1948 (2008).

10. Y. Liu, H. Tu, and S. A. Boppart, Opt. Lett. 37, 2172-2174 (2012).

11. M. Conforti and S. Trillo, Opt. Lett. 38, 3815--3818 (2013).

12.J. Fatome, C. Finot, G. Millot, A. Armaroli, and S. Trillo, Phys. Rev. X 4 , 021022 (2014).

13. C. Lecaplain, J. M. Soto-Crespo, Ph. Grelu, and C. Conti, Opt. Lett. 39, 263-266 (2014).

14. G. Xu, A. Mussot, A. Kudlinski, S. Trillo, F. Copie, and M. Conforti, Opt. Lett. 41, 2656-2659 (2016).

15. G. Xu, M. Conforti, A. Mussot, A. Kudlinski, and S. Trillo, Phys. Rev. Lett. 118, 254101 (2017).

16.J. M. Dudley, C. Finot, D. J. Richardson, and G. Millot, Nat. Phys. 3, 597603 (2007).

17. V. A. Brazhnyi, A.M. Kamchatnov, and V.V. Konotop, Phys. Rev. A 68, 035603 (2003)

18. G. Millot, S. Pitois, M. Yan, T. Hovhannisyan, A. Bendahmane, T.W. Hänsch, and N. Picqué, Nat. Photon. 10, 27-30 (2016).

19. M. Yan, P.-L. Luo, K. Iwakuni, G. Millot, T. W. Hänsch, and N. Picqué, Light: Science \& Applications 6, e17076 (2017) accepted article preview; doi: 10.1038/lsa.2017.76.

20. R. Wu, V. R. Supradeepa, C. M. Long, D. E. Leaird, and A. M. Weiner, Opt. Lett. 35, 3234-3236 (2010).

21. R. Wu, V. Torres-Company, D. E. Leaird, and A. M. Weiner, Opt. Express 21, 6045-6052 (2013).

22. R. Maher, K. Shi, L. P. Barry, J. o'Carroll, B. Kelly, R. Phelan, J. O'Gorman, and P. M. Anandarajah, Opt. Express 18, 15672-15681 (2010).

23. S. Trillo, J. S. T. Gongora, and A. Fratalocchi, Sci. Rep. 4, 7285 (2014).

24. D. Castello-Lurbe, P. Andrés, and E. Silvestre, Opt. Express 21, 2855028558 (2013).

25.D. Castello-Lurbe, N. Vermeulen, and E. Silvestre, Opt. Express 24, 26629-26645 (2016).

26. A. Zeytunyan, G. Yesayan, L. Mouradian, P. Kockaert, Ph. Emplit, F. Louradour, and A. Barthélémy, J. Eur. Opt. Soc., Rap. Pub. 4, 09009 (2009).

27.S. O. lakushev, O. V. Shulika, and I. A. Sukhoivanov, Opt. Commun. 285, 4493-4499 (2012). 


\section{Full References}

1. G. A. El, and M. A. Hofer, "Dispersive shock waves and modulation theory," Physica D 333, 11-65 (2016).

2. A. M. Kamchatnov, A. Gammal, and R. A. Kraenkel, "Dissipationless shock waves in Bose-Einstein condensates with repulsive interaction between atoms," Phys. Rev. A 69, 063605 (2004).

3. W. Wan, S. Jia, and J. W. Fleischer, "Dispersive superfluid-like shock waves in nonlinear optics," Nat. Phys. 3, 46-51 (2007).

4. M. D. Maiden, N. K. Lowman, D. V. Anderson, M. E. Schubert, and M. A. Hoefer, "Observation of dispersive shock waves, solitons, and their interactions in viscous fluid conduits," Phys. Rev. Lett. 116, 174501 (2016).

5. W. J. Tomlinson, R. H. Stolen, and A. M. Johnson, "Optical wave breaking of pulses in nonlinear optical fibers," Opt. Lett. 10, 457-459 (1985).

6. J. E. Rothenberg and D. Grischkowsky, "Observation of the formation of an optical intensity shock and wave breaking in the nonlinear propagation of pulses in optical fibers," Phys. Rev. Lett. 62, 531-534 (1989).

7. D. Anderson, M. Desaix, M. Lisak, and M. L. Quiroga-Teixeiro, "Wave breaking in nonlinear-optical fibers,".J. Opt. Soc. Am. B. 9, 1358-1361 (1992).

8. C.-J. Rosenberg, D. Anderson, M. Desaix, P. Johannisson, and M. Lisak, "Evolution of optical pulses towards wave breaking in highly nonlinear fibres," Opt. Comm. 273, 272-277 (2007).

9. C. Finot, B. Kibler, L. Provost, and S. Wabnitz, "Beneficial impact of wave-breaking for coherent continuum formation in normally dispersive nonlinear fibers,".J. Opt. Soc. Am. B. 25, 1938-1948 (2008).

10.Y. Liu, H. Tu, and S. A. Boppart, "Wave-breaking-extended fiber supercontinuum generation for high compression ratio transformlimited pulse compression," Opt. Lett. 37, 2172-2174 (2012).

11.M. Conforti and S. Trillo, "Dispersive wave emission from wave breaking", Opt. Lett. 38, 3815--3818 (2013).

12.J. Fatome, C. Finot, G. Millot, A. Armaroli, and S. Trillo, "Observation of optical undular bores in multiple four-wave mixing," Phys. Rev. X 4, 021022 (2014).

13.C. Lecaplain, J. M. Soto-Crespo, Ph. Grelu, and C. Conti, "Dissipative shock waves in all-normal-dispersion mode-locked fiber lasers," Opt. Lett. 39, 263-266 (2014).

14. G. Xu, A. Mussot, A. Kudlinski, S. Trillo, F. Copie, and M. Conforti, "Shock wave generation triggered by a weak background in optical fibers," Opt. Lett. 41, 2656-2659 (2016).

15. G. Xu, M. Conforti, A. Mussot, A. Kudlinski, and S. Trillo, "Dispersive Dam-Break Flow of a Photon Fluid," Phys. Rev. Lett. 118, 254101 (2017).

16.J. M. Dudley, C. Finot, D. J. Richardson, and G. Millot, "Self-similarity in ultrafast nonlinear optics," Nat. Phys. 3, 597-603 (2007).

17.V. A. Brazhnyi, A.M. Kamchatnov, and V.V. Konotop, "Hydrodynamic flow of expanding Bose - Einstein condensates," Phys. Rev. A 68, 035603 (2003).

18. G. Millot, S. Pitois, M. Yan, T. Hovhannisyan, A. Bendahmane, T.W. Hänsch, and N. Picqué, "Frequency-agile dual-comb spectroscopy," Nat. Photon. 10, 27-30 (2016).

19. M. Yan, P.-L. Luo, K. Iwakuni, G. Millot, T. W. Hänsch, and N. Picqué, "Mid-infrared dual-comb spectroscopy with electro-optics modulators," Light: Science \& Applications 6, e17076 (2017) accepted article preview; doi: 10.1038/Isa.2017.76.

20. R. Wu, V. R. Supradeepa, C. M. Long, D. E. Leaird, and A. M. Weiner, "Generation of very flat optical frequency combs from continuouswave lasers using cascaded intensity and phase modulators driven by tailored radio frequency waveforms," Opt. Lett. 35, 3234-3236 (2010).
21.R. Wu, V. Torres-Company, D. E. Leaird, and A. M. Weiner, "Supercontinuum-based 10-GHz flat-topped optical frequency comb generation," Opt. Express 21, 6045-6052 (2013).

22. R. Maher, K. Shi, L. P. Barry, J. o'Carroll, B. Kelly, R. Phelan, J. O'Gorman, and P. M. Anandarajah, "Implementation of a cost-effective optical comb source in a WDM-PON with $10.7 \mathrm{~Gb} / \mathrm{s}$ data to each ONU and 50km reach," Opt. Express 18, 15672-15681 (2010).

23.S. Trillo, J. S. T. Gongora, and A. Fratalocchi, "Wave instabilities in the presence of non vanishing background in nonlinear Schrödinger systems," Sci. Rep. 4, 7285 (2014).

24.D. Castello-Lurbe, P. Andrés, and E. Silvestre, "Dispersion-to-spectrum mapping in nonlinear fibers based on optical wave-breaking," Opt. Express 21, 28550-28558 (2013).

25.D. Castello-Lurbe, N. Vermeulen, and E. Silvestre, "Towards an analytical framework for tailoring supercontinuum generation", Opt. Express 24, 26629-26645 (2016).

26.A. Zeytunyan, G. Yesayan, L. Mouradian, P. Kockaert, Ph. Emplit, F. Louradour, and A. Barthélémy, "Nonlinear-dispersive similariton of passive fiber," J. Eur. Opt. Soc., Rap. Pub. 4, 09009 (2009).

27.S. O. lakushev, O. V. Shulika, and I. A. Sukhoivanov, "Passive nonlinear reshaping towards parabolic pulses in the steady-state regime in optical fibers," Opt. Commun. 285, 4493-4499 (2012). 